\title{
Identifier Extension
}

National Cancer Institute

\section{Source}

National Cancer Institute. Identifier Extension. NCI Thesaurus. Code C70904.

A character string as a unique identifier within the scope of the identifier root. 